\title{
25. List of the Chromosome Numbers in the Genus Crocus.
}

\author{
By Kôtarô Karasawa.
}

(Comm. by S. IKeno, M.I.A., Feb. 12, 1942.)

\section{Introduction}

The genus Crocus, according to Bailey (1924), comprises probably seventy-five species, most of them being found in the Mediterranean region and Asia Minor. It belongs to the Iridaceae, and is usually propagated by the corm. Scarcely more than four or five species have been commonly cultivated in our gardens. Although many other species have been grown either by botanists or by fanciers for special purposes, they are quite rarely cultivated in Japan.

Due to the abundant chromosome variations which is comparable to that of the genus Crepis, the genus Crocus has furnished excellent materials for cytological studies. As pointed out by Propach (1939), the genus shows a unique chromosome series which is ranging from 3 to 15 in haploid number, among the thousands of higher plants. Another striking fact about this genus is the occurrence of several species with haploid number as low as 3, whereas in other genera, such as Callitriche, Crepis, there are only one or two species with such remarkably low chromosome number (cf. Karasawa, 1939).

The first cytological studies in this genus were made by Himmerbaur (1926) in Crocus sativus, and also by Heitz (1926) in five species. Following these studies, Morinaga and Fukushima (1931) observed the somatic chromosomes of two species, and Brittingham (1934) reported the somatic counts of six species.

Mather (1932), in his comprehensive studies, observed the somatic chromosomes of thirty-nine species and eight varieties. My cytological studies on this genus, which were begun in 1932, the same year as Mather's, have been continued for the past ten years (Karasawa, 1932, 1933, 1935, 1937, 1939, and 1940).

Recently Propach (1939) observed the somatic chromosomes of certain species, and gave a cytological review of this genus. Pathak (1940) reported the somatic counts of twelve species and one variety, and the meiotic counts of two species. Most of his results agreed with those of the previous workers.

The object of this paper is to list my own chromosome counts of over one hundred Crocus of various species, varieties, and forms, together with the results of other investigators.

\section{Material and Methods}

The common Crocus were mostly collected from Tokyo Nôsan: a few of them were gathered from Yamato Syubyô and Takii. The other rare Crocus were obtained directly from Barr \& Sons and C.G. 
van Tubergen. One species, Crocus reticulatus, was kindly sent by the John Innes Horticultural Institution. The root-tips were mostly fixed in Navashin's fluid, some being treated with Flemming's fluid. Chloral hydrate was sometimes used in order to obtain clear figures. The somatic chromosomes were counted by staining exclusively with Heidenhain's iron-alum haematoxylin. The meiotic chromosomes were mostly observed with aceto-carmine, although a few of them were counted from permanent preparations.
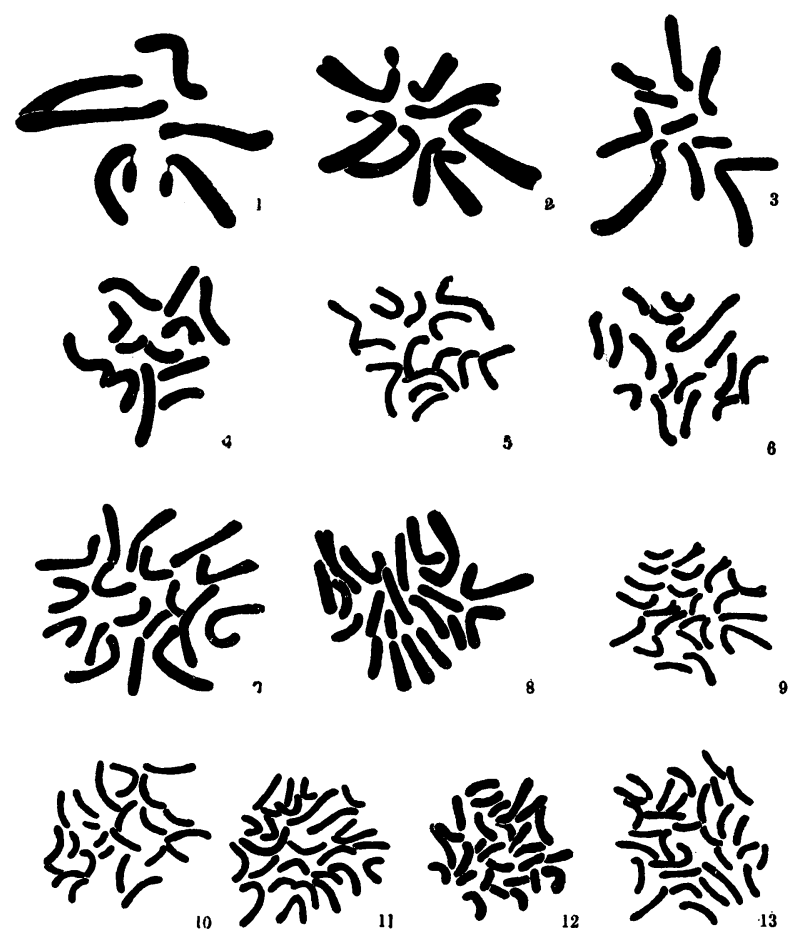

Figs. 1-13. A chromosome series in the genus Crocus. 1, C. Olivieri $(2 \mathrm{n}=6) .2, C$. aureus $(2 \mathrm{n}=8) .3$, C. cancellatus $(2 \mathrm{n}=10) .4$, C. pulchellus $(2 \mathrm{n}=12)$. 5, C. Heuffelianus $(2 \mathrm{n}=14) .6$, C. sativus $(2 \mathrm{n}=16) .7$, C. speciosus $(2 \mathrm{n}=18) .8$, C. Koralkowii $(2 \mathrm{n}=20) .9$, C. asturicus $(2 \mathrm{n}=$ 22). 10, C. Salzmannii $(2 \mathrm{n}=24)$. 11, C. Imperati $(2 \mathrm{n}=26)$. 12, C. Pestallozzae $(2 \mathrm{n}=28) . \quad 13$, C. Tournefortii $(2 \mathrm{n}=30) . \quad \times 1600$.

\section{Acknowledgment}

The expenses in connection with the present research were mostly defrayed from the Imperial Academy, to the council of which I wish to express my cordial thanks. My thanks are also due to the Society for the Promotion of Scientific and Industrial Research of Japan, from which a part of the expenses was granted. I am very much obliged to Mr. Kiyokazu Takatsu who gave me substantial encouragement. 
List of chromosome numbers

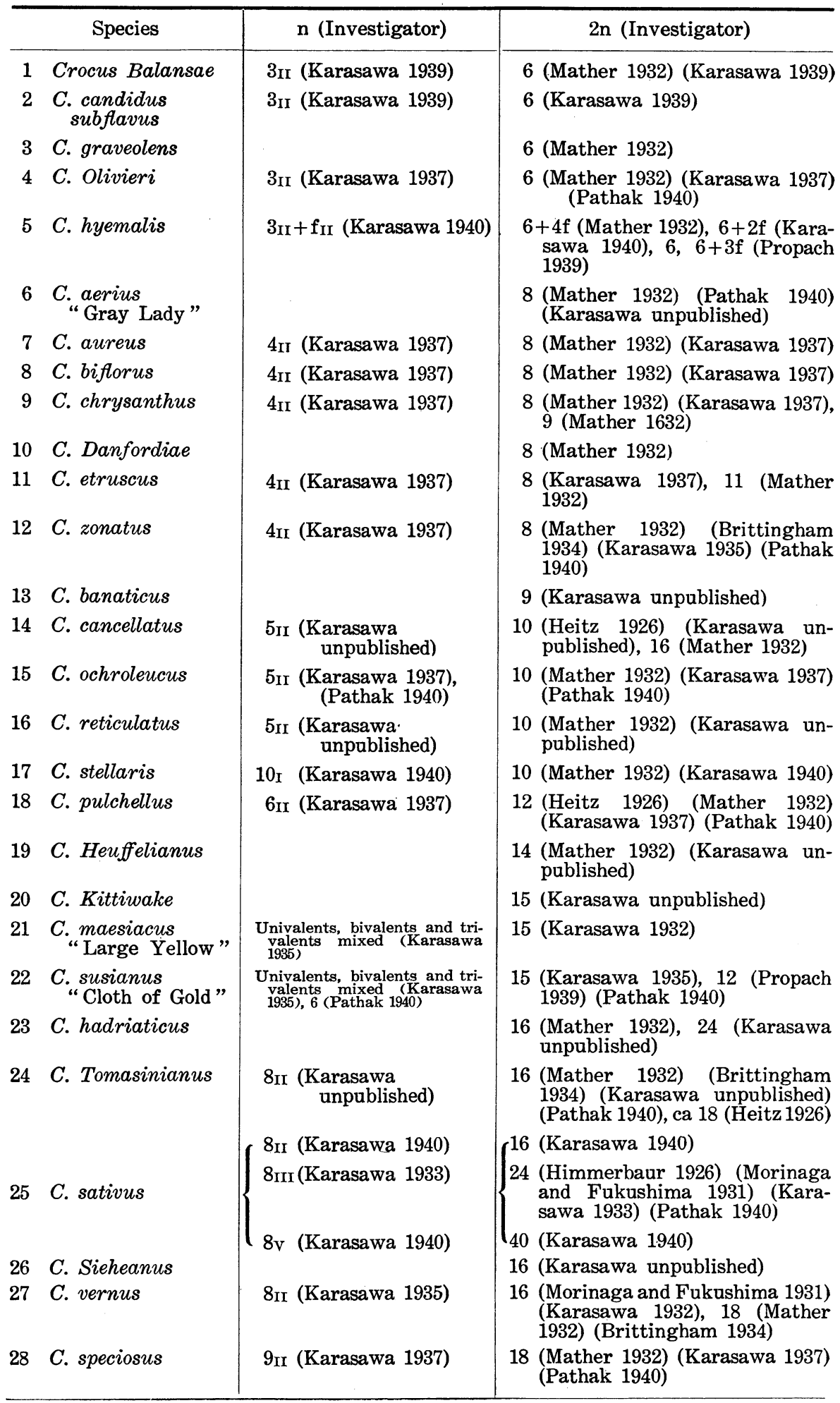




\begin{tabular}{|c|c|c|c|}
\hline & Species & n (Investigator) & 2n (Investigator) \\
\hline 29 & C. Fleischeri & 10II (Karasawa 1940) & 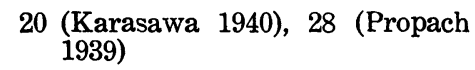 \\
\hline 30 & C. karduchorum & & 20 (Mather 1932) \\
\hline 31 & C. Korolkowii & 10II (Karasawa 1940) & $\begin{array}{l}20 \text { (Mather 1932) (Brittingham } \\
\text { 1934) (Karasawa 1940) (Pathak } \\
\text { 1940) }\end{array}$ \\
\hline 32 & C. Leichtlini & & 20 (Mather 1932) \\
\hline 33 & C. asturicus & $\begin{array}{l}\text { 11. } \\
\text { unpublished) }\end{array}$ & $\begin{array}{l}22 \text { (Mather 1932) (Karasawa un- } \\
\text { published), 22-(24) (Heitz 1926) }\end{array}$ \\
\hline 34 & C. corsicus & & $\begin{array}{l}22 \text { (Mather 1932) (Karasawa un- } \\
\text { published) }\end{array}$ \\
\hline 35 & C. dalmaticus & & 22 (Karasawa unpublished) \\
\hline 36 & C. medius & & 22 (Karasawa unpublished) \\
\hline 37 & C. Sieberi & 111I (Karasawa 1937) & 22 (Mather 1932) (Karasawa 1937) \\
\hline 38 & C. Boryi & & 24 (Mather 1932) \\
\hline 39 & C. Malyi & & 24 (Mather 1932) \\
\hline 40 & C. minimus & & 24 (Karasawa unpublished) \\
\hline 41 & C. Salzmannii & 12II (Karasawa 1940) & $24 \underset{\text { (Pathak 1940) }}{\text { (Mather 1932) (Kasawa 1940) }}$ \\
\hline 42 & C. Imperati & 13II (Karasawa 1937) & $\begin{array}{l}26 \text { (Mather 1932) } \text { (Brittingham } \\
\text { 1934) (Karasawa 1937) }\end{array}$ \\
\hline 43 & C. iridiflorus & & $\begin{array}{l}26 \text { (Mather 1932) (Karasawa un- } \\
\text { published), 24-(26) (Heitz 1926) }\end{array}$ \\
\hline 44 & $\begin{array}{l}\text { C. laevigatus } \\
\text { Fontenayii }\end{array}$ & & $\begin{array}{l}26 \text { (Mather 1932) (Karasawa un- } \\
\text { published) }\end{array}$ \\
\hline 45 & C. versicolor & $\left\{\begin{array}{c}13 \text { II (Karasawa } \\
\text { unpublished) }\end{array}\right.$ & $\left\{\begin{array}{l}26 \text { (Mather 1932) (Brittingham } \\
\text { 1934) }\end{array}\right.$ \\
\hline & & (13III (Karasawa 1937) & l39 (Karasawa 1937) \\
\hline 46 & C. longiflorus & $\begin{array}{c}\text { 14II (Karasawa } \\
\text { unpublished) }\end{array}$ & $\begin{array}{l}28 \text { (Mather 1932) (Karasawa un- } \\
\text { published) }\end{array}$ \\
\hline 47 & C. Pestallozzae & & 28 (Karasawa unpublished) \\
\hline 48 & C. Tournefortii & $\begin{array}{l}\text { 15Ir (Karasawa } \\
\text { unpublished) }\end{array}$ & 30 (Mather 1932) (Pathak 1940) \\
\hline 49 & C. nudiflorus & & $\begin{array}{l}\text { ca } 46 \text { (Mather 1932) (Karasawa } \\
\text { unpublished) }\end{array}$ \\
\hline
\end{tabular}

\begin{tabular}{c|c|c}
\hline Variety and Form & n (Investigator) & 2n (Investigator) \\
\hline I Crocus Olivieri & & 6 (Mather 1932) \\
$1 \quad$ C. Aucheri $\left.{ }^{1}\right)$ & & 14 (Mather 1932) \\
II C. aerius & \\
$2 \quad$ Celeste & 8 (Karasawa unpublished) \\
III C. aureus & & 8 (Karasawa unpublished) \\
3 maesiacus \\
4 sulphureus & 4II (Karasawa unpublished) & \\
IV C. biflorus & & 8 (Karasawa unpublished) \\
5 argenteus & & 7 (Mather 1932) (Karasawa \\
6 Barrii & & unpublished) \\
\hline
\end{tabular}

1) According to Mather, C. Aucheri may be only a variety of C. Olivieri. 


\begin{tabular}{|c|c|c|c|}
\hline \multicolumn{2}{|c|}{ Variety and Form } & n (Investigator) & \multirow{2}{*}{$\frac{\text { 2n (Investigator) }}{8 \text { (Karasawa unpublished) }}$} \\
\hline 7 & Parkinsonii & & \\
\hline 8 & pusillus & & $\begin{array}{l}8 \text { (Mather 1932) (Karasawa } \\
\text { unpublished) }\end{array}$ \\
\hline 9 & Weldeni & 5III (Karasawa unpublished) & 15 (Karasawa unpublished) \\
\hline & Weldeni albus & & 20 (Karasawa unpublished) \\
\hline \multicolumn{4}{|c|}{ V C. cancellatus } \\
\hline & albus & & 16 (Karasawa unpublished) \\
\hline \multicolumn{4}{|c|}{ VI C. chrysanthus } \\
\hline & canary bird & 5II (Karasawa unpublished) & 10 (Mather unpublished) \\
\hline & fusco-tinctus & & 10 (Mather 1932) \\
\hline & E. A. Bowles & & 8 (Karasawa unpublished) \\
\hline & E. P. Bowles & & 8 (Karasawa unpublished) \\
\hline & Snow Bunting & & 8 (Karasawa unpublished) \\
\hline VII C.Sc & alzmannii & & \\
\hline 17 & erectophyllus & 12II (Karasawa 1940) & 24 (Karasawa 1940) \\
\hline \multicolumn{4}{|c|}{ VIII C. sativus } \\
\hline & cappadocicum & & 14 (Mather 1932) \\
\hline & Elwesii & 7II +1I (Karasawa 1937) & $\begin{array}{l}15 \text { (Mather'1932) (Karasawa } \\
\text { 1937) (Pathak 1940) }\end{array}$ \\
\hline \multicolumn{4}{|c|}{ IX C. speciosus } \\
\hline & Aitchisonii & $\begin{array}{l}\text { 6II (Karasawa 1940) } \\
\text { 7II (Karasawa 1940) }\end{array}$ & $\left\{\begin{array}{l}12 \text { (Karasawa 1940) } \\
16 \text { (Karasawa 1940) } \\
14 \text { (Karasawa 1940) }\end{array}\right.$ \\
\hline & albus & 6rr (Karasawa 1940) & $\begin{array}{l}12 \text { (Mather 1932) (Karasawa } \\
\text { 1940) (Pathak 1940) }\end{array}$ \\
\hline 22 & artabir & & 14 (Karasawa 1940) \\
\hline 23 & cassiope & & 12 (Karasawa 1940) \\
\hline 24 & globosus & & 14 (Karasawa 1940) \\
\hline 25 & pollux & & 16 (Karasawa 1940) \\
\hline \multicolumn{4}{|c|}{$\mathrm{X}$ C. stellaris } \\
\hline & pallidus & 8I (Karasawa 1940) & 8 (Karasawa 1940) \\
\hline \multicolumn{4}{|c|}{ IX C. vernus } \\
\hline 27 & albus & 4II-8I (Karasawa 1940) & $\begin{array}{l}8 \text { (Mather 1932) (Karasawa } \\
\text { 1940) }\end{array}$ \\
\hline 28 & obovatus & & 19 (Mather 1932) \\
\hline 29 & $\begin{array}{l}\text { Baron van } \\
\text { Bruno }\end{array}$ & 8II (Karasawa 1935) & 16 (Karasawa 1935) \\
\hline 30 & $\begin{array}{l}\text { King of the } \\
\text { Blue }\end{array}$ & & 16 (Karasawa 1932) \\
\hline 31 & Meier Bear & & 16 (Karasawa 1935) \\
\hline 32 & Mikado & 8II (Karasawa 1935) & 16 (Karasawa 1935) \\
\hline 33 & Mont Blanc & 8II (Karasawa 1935) & 16 (Karasawa 1932) \\
\hline 34 & Hero & & 24 (Karasawa unpublished) \\
\hline 35 & Lord Fielding & 8III (Karasawa 1935) & 24 (Karasawa 1932) \\
\hline 36 & $\begin{array}{l}\text { President } \\
\text { Grant }\end{array}$ & & 24 (Karasawa 1935) \\
\hline 37 & Prince Albert & & 24 (Karasawa 1932) \\
\hline 38 & Vanguard & 8III (Karasawa unpublished) & 24 (Karasawa unpublished) \\
\hline 39 & White Lady & & 24 (Karasawa unpublished) \\
\hline 40 & Julia Culp & 8IV (Karasawa 1935) & 32 (Karasawa 1932) \\
\hline
\end{tabular}




\begin{tabular}{|c|c|c|c|}
\hline Variety & and Form & n (Investigator) & 2n (Investigator) \\
\hline 41 & $\begin{array}{l}\text { Purprea } \\
\text { Grandiflora }\end{array}$ & \multirow{22}{*}{$\begin{array}{l}\text { 10II (Karasawa unpublished) } \\
\text { 10Ir (Karasawa unpublished) }\end{array}$} & 32 (Karasawa 1935) \\
\hline 42 & Cloth of Silver & & 32 (Karasawa 1935) \\
\hline 43 & Remembrance & & 32 (Karasawa unpublished) \\
\hline 44 & Striped Beauty & & 32 (Karasawa unpublished) \\
\hline 45 & Agnes & & 20 (Karasawa unpublished) \\
\hline 46 & Albion Striped & & 20 (Karasawa 1935) \\
\hline 47 & $\begin{array}{c}\text { King of the } \\
\text { White }\end{array}$ & & 20 (Karasawa 1935) \\
\hline 48 & Maximilian & & 20 (Karasawa 1935) \\
\hline 49 & $\begin{array}{l}\text { President } \\
\text { Galivan }\end{array}$ & & 20 (Karasawa unpublished) \\
\hline 50 & Queen Victoria & & 20 (Karasawa unpublished) \\
\hline 51 & Zaxass & & 20 (Karasawa 1935) \\
\hline 52 & Pallas & & 30 (Karasawa 1935) \\
\hline 53 & Enchantress & & ca 21 (Karasawa unpublished) \\
\hline 54 & Jubilee & & ca 23 (Karasawa unpublished) \\
\hline 55 & Potter & & 25 (Karasawa unpublished) \\
\hline 56 & $\begin{array}{r}\text { Kathleen } \\
\text { Parlow }\end{array}$ & & 27 (Karasawa unpublished) \\
\hline 57 & Snowstorm & & 27 (Karasawa unpublished) \\
\hline 58 & Gladstone & & ca 28 (Karasawa unpublished) \\
\hline 59 & The Bishop & & ca 28 (Karasawa unpublished) \\
\hline 60 & Albion & & ca 29 (Karasawa unpublished) \\
\hline 61 & Albion Purple & & 29 (Karasawa unpublished) \\
\hline 62 & Excelsior & & ca 29 (Karasawa unpublished) \\
\hline
\end{tabular}

\section{Literature cited}

Bailey, L. H. (1924) Manual of Cultivated Plants. Macmillan, London. Pp. $8+851$.

Brittingham, W. H. (1934) Cytological studies on some genera of the Iridaceae. Amer. Jour. Bot., 21 : 77-82.

Heitz, E. (1926) Der Nachweis der Chromosomen. Zeit. f. Bot., 18:625-681.

Himmerbaur, W. (1926) Zur Entwicklungsgeschite von Crocus sativus. TschirchFestschrift, Leipzig. 335-350.

Karasawa, K. (1932) Karyological studies of some flowering Crocus. Bot. Mag., Tokyo. $46: 800-802$.

- (1933) On the triploidy of Crocus sativus, L. and its high sterility. Jap. Jour. Genetics, $9: 6-8$.

- (1935) Cytological studies on the cultivated Crocus. Ibid, 11:162-168.

(1937) Karyological studies in Crocus I. Jap. Jour. Bot., 9:1-15.

(1939) On the chromosomes of Crocus Balansae and Crocus candidus subflavus. Genetica, $21: 88-91$.

(1940) Karyological studies in Crocus II. Jap. Jour. Bot., 11 : 129-140.

Mather, K. (1932) Chromosome variation in Crocus I. Jour. Genetics, 26:129-142.

Morinaga, T. and Fukushima, E. (1931) Chromosome numbers of cultivated plants III. Bot. Mag., Tokyo. $45: 140-145$.

Pathak, G. N. (1940) Studies in the cytology of Crocus. Ann. of Bot., N. S. 4:227256.

Propach, H. (1939) Cytogenetik bei Zierpflanzen. (Sammelreferat). Züchter, 11:174184. 